\title{
Dark Energy and Dark Matter
}

Keith A. Olive ${ }^{1}$

${ }^{1}$ William I. Fine Theoretical Physics Institute, University of Minnesota, Minneapolis, MN 55455

A brief overview of our current understanding of abundance and properties of dark energy and dark matter is presented. A more focused discussion of supersymmetric dark matter follows. Included is a frequentist approach to the supersymmetric parameter space and consequences for the direct detection of dark matter.

\section{The Energy Density Content of the Universe}

The overall composition of the Universe can be conveniently described by the density parameter, $\Omega$, defined as the average energy density of the Universe, $\rho$, relative to the critical density needed for a spatially flat Universe, $\rho_{c}$. One of the Einstein field equations leads to the expression for the expansion rate of the Universe, which we characterize by the Hubble parameter,

$$
H^{2} \equiv\left(\frac{\dot{R}}{R}\right)^{2}=\frac{8 \pi G_{N} \rho}{3}-\frac{k}{R^{2}}+\frac{\Lambda}{3},
$$

where $R(t)$ is the cosmological scale factor and $k$ is the three-space curvature constant $(k=$ $0,+1,-1$ for a spatially flat, closed or open universe). $\Lambda$ is the cosmological constant which is assumed here to contain all contributions from the vacuum energy density. One can define a critical energy density $\rho_{c}$ such that $\rho=\rho_{c}$ for $k=0$

$$
\rho_{c}=3 H^{2} / 8 \pi G_{N} \text {. }
$$

In terms of the present value of the Hubble parameter this is,

$$
\rho_{c}=1.88 \times 10^{-29} h_{0}^{2} \mathrm{gcm}^{-3},
$$

where

$$
h_{0}=H_{0} /\left(100 \mathrm{kmMpc}^{-1} \mathrm{~s}^{-1}\right) .
$$

The cosmological density parameter is then defined by

$$
\Omega \equiv \frac{\rho}{\rho_{c}} .
$$

The composition of the Universe can be expressed by breaking down the density parameter into separate contributions,

$$
\Omega=\Omega_{r}+\Omega_{m}+\Omega_{\Lambda},
$$

for contributions from radiation, matter and a cosmological constant/vacuum with $\Omega_{\Lambda}=$ $\Lambda / 3 H^{2}$. The contribution to $\Omega_{r}$ from the cosmic microwave background (CMB) is small, of 
order $10^{-4}$. Precise determinations of the matter and vacuum contributions to $\Omega$ are obtained from the detailed power spectrum of CMB anisotropies as measured by WMAP [1]. When combined with other measurements such as high redshift supernova type Ia data [2] and baryon acoustic oscillations [3], one finds

$$
h_{0}=0.71 \pm 0.01 \quad \Omega_{0}=1.006 \pm 0.006 .
$$

WMAP data alone is sufficient for determining the individual contributions to $\Omega$ of

$$
\Omega_{m} h_{0}^{2}=0.133 \pm 0.006 \quad \Omega_{\Lambda}=0.74 \pm 0.03 .
$$

The matter content of the Universe can be further broken down as WMAP also determines the baryon density of Universe [1]

$$
\Omega_{B} h_{0}^{2}=0.0227 \pm 0.0006 .
$$

The contribution to $\Omega$ in neutrinos lies in the range

$$
0.0005<\Omega_{\nu} h_{0}^{2}<0.0076,
$$

where the lower bound is obtained from the requirement of finite neutrino masses from oscillation data and the upper bound is again derived from WMAP data in conjunction with other large scale structure data.

\section{Dark Energy}

The biggest surprise of all of the recent determinations of contributions to $\Omega$ must be the realization that there is a substantial contribution from dark energy, namely that $\Omega_{\Lambda} \neq 0$. The WMAP value for $\Omega_{\Lambda}$ is moreover consistent with determinations from supernovae data and baryon acoustic oscillations. When all data are used, one finds $\Omega_{\Lambda}=0.726 \pm 0.015$.

But now a bigger question arises: What is the physical nature of the dark energy? Different possibilities can be distinguished by their equation of state characterized by $w=p / \rho$. The equation of state parameter for radiation is simply $w_{r}=1 / 3$, whereas for matter, it is $w_{m}=0$.

The simplest solution for the dark energy remains either a cosmological constant or a constant vacuum contribution to the energy density with an equation of state, $w=-1$. This is indeed consistent with the central value determined by WMAP, which finds [1]

$$
-0.33<1+w_{0}<0.21,
$$

for the value of $w$ today (at $95 \%$ CL). The numerical value for $\Lambda$, however, is extremely small, and when written as a dimensionless constant (as $G_{N} \Lambda$ ), it is of order $10^{-123}$. This is the well known cosmological constant problem in cosmology [4].

There are, of course, other possibilities, the largest class of which is known as quintessence [5]. In this case, the dark energy may be a dynamical phenomenon described by an evolving scalar field. The energy density and pressure of a scalar field, $\phi$, with potential, $V(\phi)$, can be written as (neglecting spatial gradient terms)

$$
\begin{aligned}
\rho & =\frac{1}{2} \dot{\phi}^{2}+V(\phi) \\
p & =\frac{1}{2} \dot{\phi}^{2}-V(\phi) .
\end{aligned}
$$


When the kinetic term is small compared to the potential, $\rho \approx V$ and $p \approx-V$, and we recover the constant solution with $w=-1$. In general, however, $w_{0}$ may differ from -1 and indeed may not even be a constant. Once again, WMAP (using supernovae and BAO data) place combined limits on $w$ and its derivative with respect to redshift, $w^{\prime}$,

$$
w=-1.06 \pm 0.14 \quad w^{\prime}=0.36 \pm 0.62
$$

In short summary, we are left with the following puzzles regarding dark energy:

- There is the question of fine-tuning associated with the cosmological constant problem. Namely, we expect several contributions to the vacuum energy density

$$
\Lambda=\Lambda_{G U T}+\Lambda_{E W}+\Lambda_{Q C D} \cdots
$$

where the various contributions listed arise from possible sources such as grand unified theories $\left(G_{N} \Lambda_{G U T} \sim\left(10^{-3}\right)^{4}\right)$, the Standard Model $\left(G_{N} \Lambda_{E W} \sim\left(10^{-16}\right)^{4}\right)$, and QCD $\left(G_{N} \Lambda_{Q C D} \sim\left(10^{-20}\right)^{4}\right)$, yet sum to $10^{-123}$.

- The coincidence problem. Here, we would like to understand why $\Omega_{m}$ is within a factor of a few of $\Omega_{\Lambda}$ today. This is one of the issues addressed by quintessence models and may be probed in observations testing the possibility of variability in fundamental constants.

\section{Dark Matter}

From the quoted contributions to $\Omega$ in matter and baryons from WMAP, we can obtain the density of cold dark matter from the difference between the total matter density and the baryon density [1]

$$
\Omega_{C D M} h^{2}=0.1099 \pm 0.0062
$$

or a $2 \sigma$ range of $0.0975-0.1223$ for $\Omega_{C D M} h^{2}$.

Evidence for dark matter in the universe is available from a wide range of observational data. In addition to the results from the CMB, there is the classic evidence from galactic rotation curves [6], which indicate that nearly all spiral galaxies are embedded in a large galactic halo of dark matter leading to rather constant rotational velocities at large distances from the center of the galaxy (in contrast to the expected $v^{2} \sim 1 / r$ behavior in the absence of dark matter). Other dramatic pieces of evidence can be found in combinations of X-ray observations and weak lensing showing the superposition of dark matter (from lensing) and ordinary matter from X-ray gas [7] and from the separation of baryonic and dark matter after the collision of two galaxies as seen in the Bullet cluster [8]. For a more complete discussion see [9].

In addition to being stable (or at least very long lived), the dark matter should be both electrically and color neutral. Indeed, there are very strong constraints, forbidding the existence of stable or long lived particles which are not color and electrically neutral as these would become bound with normal matter forming anomalously heavy isotopes. The limits on the abundances, relative to hydrogen, of nuclear isotopes [10], $n / n_{H} \lesssim 10^{-15}$ to $10^{-29}$ for $1 \mathrm{GeV} \lesssim m \lesssim 1$ TeV. A strongly interacting stable relic is expected to have an abundance $n / n_{H} \lesssim 10^{-10}$ with a higher abundance for charged particles.

Unfortunately, there are no viable candidates for dark matter in the Standard Model. As baryons and neutrinos have been excluded, one is forced to go beyond the Standard Model, and 
here, I will focus on the possibilities which exist in the context of the minimal supersymmetric extension of the Standard Model (MSSM) [11]. In the MSSM, the lightest supersymmetric particle (LSP) is stable if R-parity $\left(R=-1^{3 B+L+2 s}\right)$ is unbroken. There are several possibilities in the MSSM, specifically the sneutrino with spin zero, the neutralino with spin $1 / 2$, and the gravitino with spin $3 / 2$. However, a sneutrino LSP would have relatively large coherent interactions with heavy nuclei, and experiments searching directly for the scattering of massive dark matter particles on nuclei exclude a stable sneutrino weighing between a few $\mathrm{GeV}$ and several $\mathrm{TeV}$ [12]. The possible loophole of a very light sneutrino was excluded by measurements of the invisible $Z$-boson decay rate at LEP [13]. The gravitino is a viable candidate and often predicted in models based on supergravity $[14,15]$. In this case, however, its probability for direct detection is negligible.

There are four neutralinos, each of which is a linear combination of the $R=-1$ neutral fermions [16]: the wino $\tilde{W}^{3}$, the partner of the 3 rd component of the $S U(2)_{L}$ gauge boson; the bino, $\tilde{B}$; and the two neutral Higgsinos, $\tilde{H}_{1}$ and $\tilde{H}_{2}$. The mass and composition of the LSP are determined by the gaugino masses, $M_{1}$ and $M_{2}$, the Higgs mixing mass term, $\mu$, and the ratio of the two Higgs vacuum expectation values expressed as $\tan \beta$. In general, neutralinos can be expressed as a linear combination

$$
\chi=\alpha \tilde{B}+\beta \tilde{W}^{3}+\gamma \tilde{H}_{1}+\delta \tilde{H}_{2} .
$$

The relic density of neutralinos depends on additional parameters in the MSSM beyond $M_{1}, M_{2}, \mu$, and $\tan \beta$. These include the sfermion masses, $m_{\tilde{f}}$ and the Higgs pseudo-scalar mass, $m_{A}$. To determine the relic density it is necessary to obtain the general annihilation cross-section for neutralinos. In much of the parameter space of interest, the LSP is a bino and the annihilation proceeds mainly through sfermion exchange.

In its generality, the MSSM has over 100 undetermined parameters.There are good arguments based on grand unification [17] and supergravity [18] which lead to a strong reduction in the number of parameters. I will assume several unification conditions placed on the supersymmetric parameters. In all models considered, the gaugino masses are assumed to be unified at the GUT scale with value, $m_{1 / 2}$, as are the trilinear couplings with value $A_{0}$. Also common to all models considered here is the unification of all soft scalar masses set equal to $m_{0}$ at the GUT scale. With this set of boundary conditions at the GUT scale, we can use the radiative electroweak symmetry breaking conditions by specifying $\tan \beta$, and the mass, $M_{Z}$, to predict the values of $\mu$ and Higgs pseudoscalar mass, $m_{A}$. The sign of $\mu$ remains free. This class of models is often referred to as the constrained MSSM (CMSSM) [19, 20, 21, 22, 23]. In the CMSSM, the solutions for $\mu$ generally lead to a lightest neutralino which is very nearly a pure $\tilde{B}$.

I note that that while the name CMSSM is often used synonymously with mSUGRA, for minimal supergravity $[18,24]$. The latter however, has two additional constraints: $m_{3 / 2}=m_{0}$ and $B_{0}=A_{0}-m_{0}$. The former sets the unification scale scalar masses equal to the gravitino mass. This condition often results in a gravitino LSP [14]. The latter condition sets a relation between the supersymmetry breaking bilinear, trilinear and scalar mass terms. Because of this condition, $\tan \beta$ is no longer a free parameter, but must be solved for through the radiative electroweak symmetry breaking relations.

In Fig. 1, an example of the renormalization group running of the mass parameters in the CMSSM is shown. Here, we have chosen $m_{1 / 2}=250 \mathrm{GeV}, m_{0}=100 \mathrm{GeV}, \tan \beta=3, A_{0}=0$, and $\mu<0$. Indeed, it is rather amazing that from so few input parameters, all of the masses of 
the supersymmetric particles can be determined. The characteristic features that one sees in the figure, are for example, that the colored sparticles are typically the heaviest in the spectrum. This is due to the large positive correction to the masses due to $\alpha_{3}$ in the RGE's. Also, one finds that the $\widetilde{B}$, is typically the lightest sparticle. But most importantly, notice that one of the Higgs mass $^{2}$, goes negative triggering electroweak symmetry breaking [25]. (The negative sign in the figure refers to the sign of the mass squared, even though it is the mass of the sparticles which is depicted.)

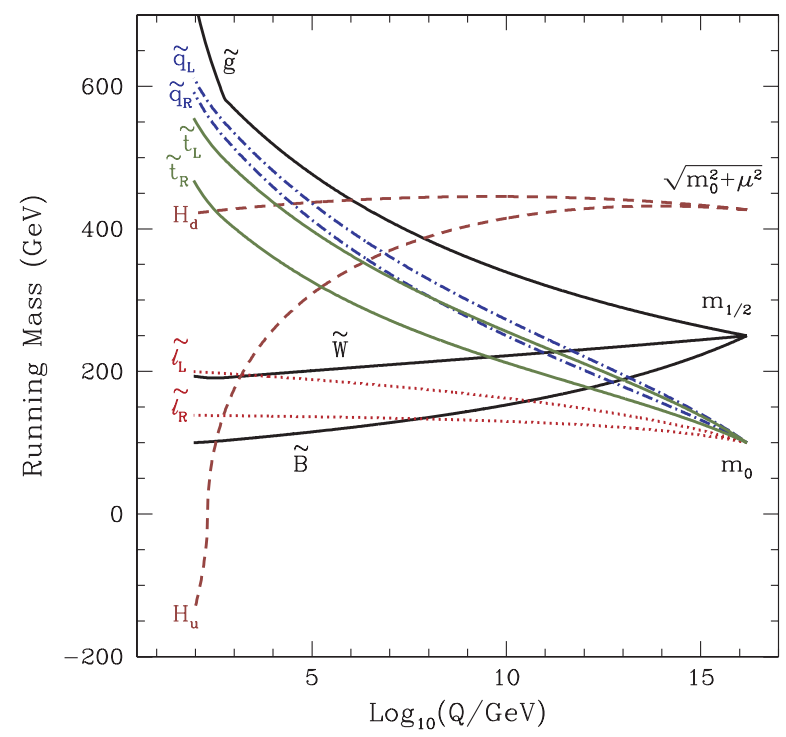

Figure 1: RG evolution of the mass parameters in the CMSSM. I thank Toby Falk for providing this figure.

For given values of $\tan \beta, A_{0}$, and $\operatorname{sgn}(\mu)$, the regions of the CMSSM parameter space that yield an acceptable relic density and satisfy the other phenomenological constraints may be displayed in the $\left(m_{1 / 2}, m_{0}\right)$ plane. In Fig. 2a, the light shaded region corresponds to that portion of the CMSSM plane with $\tan \beta=10, A_{0}=0$, and $\mu>0$ such that the computed relic density yields the WMAP value given in eq. (16) [22]. The bulk region at relatively low values of $m_{1 / 2}$ and $m_{0}$, tapers off as $m_{1 / 2}$ is increased. At higher values of $m_{0}$, annihilation cross sections are too small to maintain an acceptable relic density and $\Omega_{\chi} h^{2}$ is too large. Although sfermion masses are also enhanced at large $m_{1 / 2}$ (due to RGE running), co-annihilation processes between the LSP and the next lightest sparticle (in this case the $\tilde{\tau}$ ) enhance the annihilation cross section and reduce the relic density. This occurs when the LSP and NLSP are nearly degenerate in mass. The dark shaded region has $m_{\tilde{\tau}}<m_{\chi}$ and is excluded. The effect of coannihilations is to create an allowed band about $25-50 \mathrm{GeV}$ wide in $m_{0}$ for $m_{1 / 2} \lesssim 950 \mathrm{GeV}$, or $m_{1 / 2} \lesssim 400$ $\mathrm{GeV}$, which tracks above the $m_{\tilde{\tau}_{1}}=m_{\chi}$ contour [26].

Also shown in Fig. 2a are the relevant phenomenological constraints. These include the LEP lower limits on the chargino mass: $m_{\chi^{ \pm}}>104 \mathrm{GeV}$ [27] and on the Higgs mass: $m_{h}>$ $114 \mathrm{GeV}$ [28]. FeynHiggs [29] is used for the calculation of $m_{h}$. The Higgs limit imposes important constraints, principally on $m_{1 / 2}$ and particularly at low $\tan \beta$. Another constraint 
is the requirement that the branching ratio for $b \rightarrow s \gamma$ be consistent with the experimental measurements [30]. These measurements agree with the Standard Model, and therefore provide bounds on MSSM particles [31], such as the chargino and charged Higgs bosons, in particular. The constraint imposed by measurements of $b \rightarrow s \gamma$ also exclude small values of $m_{1 / 2}$. Finally, there are regions of the $\left(m_{1 / 2}, m_{0}\right)$ plane that are favored by the BNL measurement [32] of the anomalous magnetic moment of the muon or $g_{\mu}-2$. Here, we assume the Standard Model calculation [33] of $g_{\mu}-2$, and indicate by dashed and solid lines the contours of $1-$ and 2- $\sigma$ level deviations induced by supersymmetry.

At larger $m_{1 / 2}, m_{0}$ and $\tan \beta$, the relic neutralino density may be reduced by rapid annihilation through direct-channel $H, A$ Higgs bosons, as seen in Fig. 2(b) [19, 21]. Finally, the relic density can again be brought down into the WMAP range at large $m_{0}$ (not shown in Fig. 2), in the 'focus-point' region close the boundary where electroweak symmetry breaking ceases to be possible and the lightest neutralino $\chi$ acquires a significant higgsino component [34].

As seen in Fig. 2, the relic density constraint is compatible with relatively large values of $m_{1 / 2}$ and $m_{0}$. However, all values of $m_{1 / 2}$ and $m_{0}$ are not equally viable when the available phenomenological and cosmological constraints are taken into account. A global likelihood analysis enables one to pin down the available parameter space in the CMSSM. One can avoid the dependence on priors by performing a pure likelihood analysis as in [35], or a purely $\chi^{2}$-based fit as done in [36, 37]. Here, the results from one such analysis [38, 39] is presented, which used a Markov-Chain Monte Carlo (MCMC) technique to explore efficiently the likelihood function in the parameter space of the CMSSM. A full list of the observables and the values assumed for them in this global analysis are given in [37], as updated in [38, 39].

The best fit point is shown in Fig. 3, which also displays contours of the $\Delta \chi^{2}$ function in the CMSSM. The parameters of the best-fit CMSSM point are $m_{0}=60 \mathrm{GeV}, m_{1 / 2}=310 \mathrm{GeV}$, $A_{0}=130 \mathrm{GeV}, \tan \beta=11$ and $\mu=400 \mathrm{GeV}$, yielding the overall $\chi^{2} / \mathrm{N}_{\mathrm{dof}}=20.6 / 19(36 \%$ probability) and nominally $M_{h}=114.2 \mathrm{GeV}$ [39]. The best-fit point is in the coannihilation region of the $\left(m_{0}, m_{1 / 2}\right)$ plane. The C.L. contours extend to slightly larger values of $m_{0}$ in the CMSSM. However, the qualitative features of the $\Delta \chi^{2}$ contours indicate a preference for small $m_{0}$ and $m_{1 / 2}$. It was found in [38] that the focus-point region was disfavored at beyond the 95\% C.L. in the CMSSM. We see in Fig. 3 that this region is disfavored at the level $\Delta \chi^{2} \sim 8$ in the CMSSM.

As noted above, there are several important cosmological and phenomenological constraints on the supersymmetric parameter space. Improvements in sensitivity have made it possible for direct detection experiments $[40,41]$ to be competitive as well. The elastic cross section for $\chi$ scattering on a nucleus can be decomposed into a scalar (spin-independent) and a spindependent part. Each of these can be written in terms of the cross sections for elastic scattering off individual nucleons. The scalar part of the cross section can be written as

$$
\sigma_{\mathrm{SI}}=\frac{4 m_{r}^{2}}{\pi}\left[Z f_{p}+(A-Z) f_{n}\right]^{2},
$$

where $m_{r}$ is the $\chi$-nuclear reduced mass and

$$
\frac{f_{N}}{m_{N}}=\sum_{q=u, d, s} f_{T_{q}}^{(N)} \frac{\alpha_{3 q}}{m_{q}}+\frac{2}{27} f_{T G}^{(N)} \sum_{q=c, b, t} \frac{\alpha_{3 q}}{m_{q}},
$$

for $N=$ p or $\mathrm{n}$. The parameters $f_{T_{q}}^{(N)}$ are defined by

$$
m_{N} f_{T_{q}}^{(N)} \equiv\left\langle N\left|m_{q} \bar{q} q\right| N\right\rangle \equiv m_{q} B_{q}^{(N)}
$$


and the $\alpha_{3 q}$ contain the individual quark-neutralino scattering cross sections, see [42, 43, 44] for further details regarding the calculation of the cross section.

The elastic scattering of neutralinos on nucleons is very sensitive to the strangeness contribution to the nucleon mass and can be characterized by the parameter, $y$, which is also related to the $\pi$-nucleon sigma term $\Sigma_{\pi_{N}}$ by

$$
y \equiv \frac{2 B_{s}}{B_{u}+B_{d}}=1-\sigma_{0} / \Sigma_{\pi_{N}}
$$

where $\sigma_{0}$ is the change in the nucleon mass due to nonzero $u$ and $d$ masses and is estimated from octet baryon mass differences to be $\sigma_{0}=36 \mathrm{MeV}$ [45], and the latest determination of $\Sigma_{\pi_{N}}=64 \mathrm{MeV}$. The effect of varying these assumptions are discussed in the context of the CMSSM in $[43,44]$. Lattice calculations are now reaching the stage where they may also provide useful information on $\Sigma_{\pi N}$ [46], and a recent analysis would suggest a lower value $\Sigma_{\pi N} \lesssim 40$ [47].

In panel (a) of Fig. 4 the spin-independent neutralino-nucleon elastic scattering cross sections are shown as functions of neutralino mass for the regions of Fig. 2a that are cosmologically viable (i.e., those where the upper limit on the relic density of neutralinos is respected), and are not excluded by constraints from colliders. Here, however, parameter values corresponding to the focus point at high $m_{0}$ are also included. Also plotted are the limits on the spin-independent cross section from CDMS II [40] (solid black line) and XENON10 [41] (solid red line), as well as the sensitivities projected for XENON100 [49] (or a similar 100-kg liquid noble-gas detector such as LUX, dashed red line) and SuperCDMS at the Soudan Mine [50] (dashed black line).

There are two distinct regions in the $\left(m_{\chi}, \sigma\right)$ plane, that arising from the focus-point region at $m_{\chi} \lesssim 150 \mathrm{GeV}$ and relatively large $\sigma$, and that from the coannihilation strip. In the coannihilation strip, $50 \mathrm{GeV}<m_{\chi}<400 \mathrm{GeV}$, where the lower limit on $m_{\chi}$ is a result of the LEP constraint on the chargino mass, and the upper limit on $m_{\chi}$ corresponds to the endpoint of the coannihilation strip for $\tan \beta=10$. In contrast, the end point of the focus-point region shown is due only to the cut-off $m_{0}<2 \mathrm{TeV}$ that has been assumed. In addition, for $m_{1 / 2} \lesssim 380 \mathrm{GeV}$ in the coannihilation strip $\left(m_{\chi} \lesssim 160 \mathrm{GeV}\right)$, the nominal calculated mass of the lighter scalar MSSM Higgs boson is less than the LEP lower bound. These points are indicated by lighter shadings.

The choices $\tan \beta=10$ and $A_{0}=0$ do not yield viable direct detection cross sections that are completely representative of the range of possibilities within the CMSSM. Therefore, in Fig. 4b, we show CMSSM spin-independent neutralino-nucleon cross section, as obtained in a scan over all CMSSM parameters with $5 \leq \tan \beta \leq 55,100 \leq m_{1 / 2} \leq 2000 \mathrm{GeV}, 0 \mathrm{GeV}$ $\leq m_{0} \leq 2000 \mathrm{GeV}$, and $-3 m_{1 / 2} \leq A_{0} \leq 3 m_{1 / 2}$ [48]. We also allow both positive and negative $\mu$, except for large $\tan \beta>30$, where convergence becomes difficult in the $\mu<0$ case. At low $m_{\chi}<300 \mathrm{GeV}$, cross sections generally exceed $10^{-9} \mathrm{pb}$, and the largest scalar cross sections, which occur for $m_{\chi} \sim 100 \mathrm{GeV}$, are already excluded by CDMS II [40] and/or XENON10 [41]. These exclusions occur primarily in the focus-point region at large $\tan \beta$. On the other hand, for $m_{\chi} \gtrsim 400 \mathrm{GeV}$ scalar cross sections are well below $10^{-9} \mathrm{pb}$, and come from the coannihilation strip or the rapid-annihilation funnel that appears at large $\tan \beta$ in the CMSSM. The effective cross sections shown are suppressed for points with $\Omega_{\chi} \ll \Omega_{C D M}$, and there may be cancellations at larger $m_{\chi}$ that suppress the cross sections substantially. These regions of parameter space will not be probed by direct detection experiments in the near future [49, 50].

Finally, the frequentist analysis described above can also be used to predict the neutralinonucleon elastic scattering cross section [39]. The value of $\sigma_{p}^{\mathrm{SI}}$ shown in Fig. 5a is calculated 
assuming a $\pi$-N scattering $\sigma$ term $\Sigma_{N}=64 \mathrm{MeV}$. We see in Fig. 5 that values of the $\tilde{\chi}_{1}^{0}$-proton cross section $\sigma_{p}^{\text {SI }} \sim 10^{-8} \mathrm{pb}$ are expected in the CMSSM, and that much larger values seem quite unlikely. The $2 \mathrm{D} \chi^{2}$ function is shown in Fig. $5 \mathrm{~b}$.

\section{Acknowledgments}

This work was supported in part by DOE grant DE-FG02-94ER-40823.

\section{References}

[1] J. Dunkley et al. [WMAP Collaboration], Astrophys. J. Suppl. 180, 306 (2009) [arXiv:0803.0586 [astroph]]; E. Komatsu et al. [WMAP Collaboration], Astrophys. J. Suppl. 180, 330 (2009) [arXiv:0803.0547 [astro-ph]].

[2] A.G. Riess et al., A. J. 116, 1009 (1998); P. Garnavich et al., Astrophys. J. 509, 74 (1998); S. Perlmutter et al., Ap. J. 517, 565 (1999); A. G. Riess et al., Ap. J. 560 (2001) 49; J.L. Tonry et al., Ap. J. 594, 1 (2003); A.G. Riess et al., Astrophys. J. 659, 98 (2007); P. Astier et al. [The SNLS Collaboration], Astron. Astrophys. 447, 31 (2006) [arXiv:astro-ph/0510447].

[3] D. Eisenstein et al., Astrophys. J. 633, 560 (2005); C. Blake et al., Mon. Not. Roy. Astr. Soc. 374, 1527 (2007); W. J. Percival, et al., Mon. Not. Roy. Astron. Soc. 381, 1053 (2007); M. Kowalski et al., Astrophys. J. 686, 749 (2008).

[4] S. Weinberg, Rev. Mod. Phys. 61, 1 (1989); S. M. Carroll, Living Rev. Rel. 4, 1 (2001) [arXiv:astroph/0004075]; P. J. E. Peebles and B. Ratra, Rev. Mod. Phys. 75, 559 (2003) [arXiv:astro-ph/0207347].

[5] I. Zlatev, L. M. Wang and P. J. Steinhardt, Phys. Rev. Lett. 82, 896 (1999) [arXiv:astro-ph/9807002]; C. Armendariz-Picon, V. F. Mukhanov and P. J. Steinhardt, Phys. Rev. D 63, 103510 (2001) [arXiv:astro$\mathrm{ph} / 0006373]$.

[6] S. M. Faber and J. J. Gallagher, Ann. Rev. Astron. Astrophys. 17 (1979) 135; A. Bosma, Ap. J. 86 (1981) 1825; V. C. Rubin, W. K. Ford and N. Thonnard, Ap. J. 238 (1980) 471; V. C. Rubin, D. Burstein, W. K. Ford and N. Thonnard, Ap. J. 289 (1985) 81; T. S. Van Albada and R. Sancisi, Phil. Trans. R. Soc. Land. A320 (1986) 447; M. Persic and P. Salucci, Ap. J. Supp. 99 (1995) 501; M. Persic, P. Salucci, and F. Stel, MNRAS 281 (1996) $27 \mathrm{P}$.

[7] D. Wittman, et al., Astrophys. J. 643, 128 (2006) [arXiv:astro-ph/0507606].

[8] D. Clowe, et al., Astrophys. J. 648 (2006) L109 [arXiv:astro-ph/0608407].

[9] K. A. Olive, arXiv:astro-ph/0301505.

[10] J. Rich, M. Spiro and J. Lloyd-Owen, Phys.Rep. 151 (1987) 239; T. Yamagata, Y. Takamori and H. Utsunomiya, Phys. Rev. D 47 (1993) 1231; T. K. Hemmick et al., Phys. Rev. D41 (1990) 2074; P. F. Smith, Contemp. Phys. 29 (1998) 159; D. Javorsek, D. Elmore, E. Fischbach, D. Granger, T. Miller, D. Oliver and V. Teplitz, Phys. Rev. D 65, 072003 (2002).

[11] P. Fayet, Phys. Lett. B64 (1976) 159; Phys. Lett. B69 (1977) 489; Phys. Lett. B84 (1979) 416; H.E. Haber and G.L. Kane, Phys.Rep. 117 (1985) 75.

[12] T. Falk, K. A. Olive and M. Srednicki, Phys. Lett. B 339, 248 (1994) [arXiv:hep-ph/9409270]; C. Arina and N. Fornengo, JHEP 0711, 029 (2007) [arXiv:0709.4477 [hep-ph]].

[13] LEP Collaborations, ALEPH, DELPHI, L3 and OPAL, LEP Electroweak Working Group, SLD Electroweak Group and SLD Heavy Flavor Group, hep-ex/0312023.

[14] J. R. Ellis, K. A. Olive, Y. Santoso and V. C. Spanos, Phys. Lett. B 573 (2003) 162 [arXiv:hep-ph/0305212]; J. R. Ellis, K. A. Olive, Y. Santoso and V. C. Spanos, Phys. Rev. D 70 (2004) 055005 [arXiv:hep$\mathrm{ph} / 0405110]$

[15] J. R. Ellis, K. A. Olive, Y. Santoso and V. C. Spanos, Phys. Lett. B 588 (2004) 7 [arXiv:hep-ph/0312262]; J. L. Feng, A. Rajaraman and F. Takayama, Phys. Rev. Lett. 91 (2003) 011302 [arXiv:hep-ph/0302215]; J. L. Feng, S. Su and F. Takayama, Phys. Rev. D 70 (2004) 075019 [arXiv:hep-ph/0404231]. 
[16] J. Ellis, J.S. Hagelin, D.V. Nanopoulos, K.A. Olive and M. Srednicki, Nucl. Phys. B 238 (1984) 453; see also H. Goldberg, Phys. Rev. Lett. 50 (1983) 1419.

[17] J. R. Ellis, S. Kelley and D. V. Nanopoulos, Phys. Lett. B 249 (1990) 441 and Phys. Lett. B 260 (1991) 131; U. Amaldi, W. de Boer and H. Furstenau, Phys. Lett. B 260 (1991) 447; C. Giunti, C. W. Kim and U. W. Lee, Mod. Phys. Lett. A 6 (1991) 1745.

[18] For reviews, see: H. P. Nilles, Phys. Rep. 110 (1984) 1; A. Brignole, L. E. Ibanez and C. Munoz, arXiv:hepph/9707209, published in Perspectives on supersymmetry, ed. G. L. Kane, pp. 125-148.

[19] M. Drees and M. M. Nojiri, Phys. Rev. D 47 (1993) 376 [arXiv:hep-ph/9207234]; H. Baer and M. Brhlik, Phys. Rev. D 53 (1996) 597 [arXiv:hep-ph/9508321]; Phys. Rev. D 57 (1998) 567 [arXiv:hep-ph/9706509]; H. Baer, M. Brhlik, M. A. Diaz, J. Ferrandis, P. Mercadante, P. Quintana and X. Tata, Phys. Rev. D 63 (2001) 015007 [arXiv:hep-ph/0005027]; A. B. Lahanas, D. V. Nanopoulos and V. C. Spanos, Mod. Phys. Lett. A 16 (2001) 1229 [arXiv:hep-ph/0009065].

[20] J. R. Ellis, T. Falk, K. A. Olive and M. Schmitt, Phys. Lett. B 388 (1996) 97 [arXiv:hep-ph/9607292]; Phys. Lett. B 413 (1997) 355 [arXiv:hep-ph/9705444]; J. R. Ellis, T. Falk, G. Ganis, K. A. Olive and M. Schmitt, Phys. Rev. D 58 (1998) 095002 [arXiv:hep-ph/9801445]; V. D. Barger and C. Kao, Phys. Rev. D 57 (1998) 3131 [arXiv:hep-ph/9704403]; J. R. Ellis, T. Falk, G. Ganis and K. A. Olive, Phys. Rev. D 62 (2000) 075010 [arXiv:hep-ph/0004169]; V. D. Barger and C. Kao, Phys. Lett. B 518 (2001) 117 [arXiv:hep-ph/0106189]; L. Roszkowski, R. Ruiz de Austri and T. Nihei, JHEP 0108 (2001) 024 [arXiv:hepph/0106334]; A. B. Lahanas and V. C. Spanos, Eur. Phys. J. C 23 (2002) 185 [arXiv:hep-ph/0106345]; A. Djouadi, M. Drees and J. L. Kneur, JHEP 0108 (2001) 055 [arXiv:hep-ph/0107316]; U. Chattopadhyay, A. Corsetti and P. Nath, Phys. Rev. D 66 (2002) 035003 [arXiv:hep-ph/0201001]; J. R. Ellis, K. A. Olive and Y. Santoso, New Jour. Phys. 4 (2002) 32 [arXiv:hep-ph/0202110]; H. Baer, C. Balazs, A. Belyaev, J. K. Mizukoshi, X. Tata and Y. Wang, JHEP 0207 (2002) 050 [arXiv:hep-ph/0205325]; R. Arnowitt and B. Dutta, arXiv:hep-ph/0211417.

[21] J. R. Ellis, T. Falk, G. Ganis, K. A. Olive and M. Srednicki, Phys. Lett. B 510 (2001) 236 [arXiv:hep$\mathrm{ph} / 0102098]$.

[22] J. R. Ellis, K. A. Olive, Y. Santoso and V. C. Spanos, Phys. Lett. B 565 (2003) 176 [arXiv:hep-ph/0303043].

[23] H. Baer and C. Balazs, JCAP 0305, 006 (2003) [arXiv:hep-ph/0303114]; A. B. Lahanas and D. V. Nanopoulos, Phys. Lett. B 568, 55 (2003) [arXiv:hep-ph/0303130]; U. Chattopadhyay, A. Corsetti and P. Nath, Phys. Rev. D 68, 035005 (2003) [arXiv:hep-ph/0303201]; . Munoz, Int. J. Mod. Phys. A 19, 3093 (2004) [arXiv:hep-ph/0309346].

[24] R. Barbieri, S. Ferrara and C.A. Savoy, Phys. Lett. 119B (1982) 343.

[25] L.E. Ibáñez and G.G. Ross, Phys. Lett. B110 (1982) 215;

L.E. Ibáñez, Phys. Lett. B118 (1982) 73;

J. Ellis, D.V. Nanopoulos and K. Tamvakis, Phys. Lett. B121 (1983) 123;

J. Ellis, J. Hagelin, D.V. Nanopoulos and K. Tamvakis, Phys. Lett. B125 (1983) 275;

L. Alvarez-Gaumé, J. Polchinski, and M. Wise, Nucl. Phys. B221 (1983) 495.

[26] J. Ellis, T. Falk, and K.A. Olive, Phys. Lett. B444 (1998) 367 [arXiv:hep-ph/9810360]; J. Ellis, T. Falk, K.A. Olive, and M. Srednicki, Astr. Part. Phys. 13 (2000) 181 [Erratum-ibid. 15 (2001) 413] [arXiv:hep$\mathrm{ph} / 9905481]$.

[27] Joint LEP 2 Supersymmetry Working Group, Combined LEP Chargino Results, up to 208 GeV, http://lepsusy. web.cern.ch/lepsusy/www/inos_moriond01/charginos_pub.html.

[28] R. Barate et al. [ALEPH, DELPHI, L3, OPAL Collaborations: the LEP Working Group for Higgs boson searches], Phys. Lett. B 565, 61 (2003) [arXiv:hep-ex/0306033]; D. Zer-Zion, Prepared for 32nd International Conference on High-Energy Physics (ICHEP 04), Beijing, China, 16-22 Aug 2004; LHWG-NOTE-2004-01, ALEPH-2004-008, DELPHI-2004-042, L3-NOTE-2820, OPAL-TN-744, http://lephiggs . web. cern.ch/LEPHIGGS/papers/August2004_MSSM/index.html.

[29] S. Heinemeyer, W. Hollik and G. Weiglein, Comput. Phys. Commun. 124 (2000) 76 [arXiv:hepph/9812320]; S. Heinemeyer, W. Hollik and G. Weiglein, Eur. Phys. J. C 9 (1999) 343 [arXiv:hepph/9812472]; G. Degrassi, S. Heinemeyer, W. Hollik, P. Slavich and G. Weiglein, Eur. Phys. J. C 28 (2003) 133 [arXiv:hep-ph/0212020].; M. Frank, T. Hahn, S. Heinemeyer, W. Hollik, H. Rzehak and G. Weiglein, JHEP 0702 (2007) 047 [arXiv:hep-ph/0611326]; http://www.feynhiggs.de/ 
[30] S. Chen et al. [CLEO Collaboration], Phys. Rev. Lett. 87 (2001) 251807 [arXiv:hep-ex/0108032]; P. Koppenburg et al. [Belle Collaboration], Phys. Rev. Lett. 93 (2004) 061803 [arXiv:hep-ex/0403004]. B. Aubert et al. [BaBar Collaboration], arXiv:hep-ex/0207076; E. Barberio et al. [Heavy Flavor Averaging Group (HFAG)], arXiv:hep-ex/0603003.

[31] C. Degrassi, P. Gambino and G. F. Giudice, JHEP 0012 (2000) 009 [arXiv:hep-ph/0009337], as implemented by P. Gambino and G. Ganis.

[32] [The Muon g-2 Collaboration], Phys. Rev. Lett. 92 (2004) 161802, hep-ex/0401008; G. Bennett et al. [The Muon g-2 Collaboration], Phys. Rev. D 73 (2006) 072003 [arXiv:hep-ex/0602035].

[33] M. Davier, S. Eidelman, A. Höcker and Z. Zhang, Eur. Phys. J. C 31 (2003) 503, hep-ph/0308213; see also M. Knecht, Lect. Notes Phys. 629, 37 (2004) [arXiv:hep-ph/0307239]; K. Melnikov and A. Vainshtein, Phys. Rev. D70 (2004) 113006 [arXiv:hep-ph/0312226]; J. F. de Troconiz and F. J. Yndurain, Phys. Rev. D 71, 073008 (2005) [arXiv:hep-ph/0402285]; M. Passera, arXiv:hep-ph/0411168; K. Hagiwara, A. Martin, D. Nomura and T. Teubner, Phys. Lett. B 649 (2007) 173 [arXiv:hep-ph/0611102]; M. Davier, Nucl. Phys. Proc. Suppl. 169, 288 (2007) [arXiv:hep-ph/0701163]; F. Jegerlehner, Acta Phys. Polon. B 38, 3021 (2007) [arXiv:hep-ph/0703125]; J. Miller, E. de Rafael and B. Roberts, Rept. Prog. Phys. 70 (2007) 795 [arXiv:hep-ph/0703049]; S. Eidelman, talk given at the ICHEP06, Moscow, July 2006, see: http://ichep06.jinr.ru/reports/333_6s1_9p30_Eidelman.pdf; M. Davier, A. Hoecker, B. Malaescu, C. Z. Yuan and Z. Zhang, arXiv:0908.4300 [hep-ph].

[34] J. L. Feng, K. T. Matchev and T. Moroi, Phys. Rev. D 61 (2000) 075005 [arXiv:hep-ph/9909334].

[35] J. R. Ellis, K. A. Olive, Y. Santoso and V. C. Spanos, Phys. Rev. D 69 (2004) 095004 [arXiv:hep$\mathrm{ph} / 0310356]$.

[36] J. R. Ellis, S. Heinemeyer, K. A. Olive, A. M. Weber and G. Weiglein, JHEP 0708, 083 (2007) [arXiv:0706.0652 [hep-ph]].

[37] O. Buchmueller et al., Phys. Lett. B 657 (2007) 87 [arXiv:0707.3447 [hep-ph]].

[38] O. Buchmueller et al., JHEP 0809 (2008) 117 [arXiv:0808.4128 [hep-ph]].

[39] O. Buchmueller et al., Eur. Phys. J. C 64, 391 (2009) [arXiv:0907.5568 [hep-ph]].

[40] Z. Ahmed et al. [CDMS Collaboration], Phys. Rev. Lett. 102, 011301 (2009) [arXiv:0802.3530 [astro-ph]].

[41] J. Angle et al. [XENON Collaboration], Phys. Rev. Lett. 100, 021303 (2008) [arXiv:0706.0039 [astro-ph]].

[42] J. Ellis, A. Ferstl and K. A. Olive, Phys. Lett. B 481, 304 (2000) [arXiv:hep-ph/0001005]; J. Ellis, A. Ferstl and K. A. Olive, Phys. Rev. D 63, 065016 (2001) [arXiv:hep-ph/0007113]; J. R. Ellis, A. Ferstl and K. A. Olive, Phys. Lett. B 532, 318 (2002) [arXiv:hep-ph/0111064].

[43] J. R. Ellis, K. A. Olive, Y. Santoso and V. C. Spanos, Phys. Rev. D 71, 095007 (2005) [arXiv:hep$\mathrm{ph} / 0502001]$.

[44] J. R. Ellis, K. A. Olive and C. Savage, Phys. Rev. D 77, 065026 (2008) [arXiv:0801.3656 [hep-ph]].

[45] B. Borasoy and U. G. Meissner, Annals Phys. 254, 192 (1997) [arXiv:hep-ph/9607432]; J. Gasser, H. Leutwyler and M. E. Sainio, Phys. Lett. B 253, 252 (1991); M. Knecht, PiN Newslett. 15, 108 (1999) [arXiv:hep-ph/9912443]; M. E. Sainio, PiN Newslett. 16, 138 (2002) [arXiv:hep-ph/0110413].

[46] R. D. Young and A. W. Thomas, arXiv:0901.3310 [hep-lat].

[47] J. Giedt, A. W. Thomas and R. D. Young, arXiv:0907.4177 [hep-ph].

[48] J. Ellis, K. A. Olive and P. Sandick, arXiv:0905.0107 [hep-ph].

[49] E. Aprile, L. Baudis and f. t. X. Collaboration, arXiv:0902.4253 [astro-ph.IM].

[50] SuperCDMS Development Project, Fermilab Proposal 0947, October 2004. 

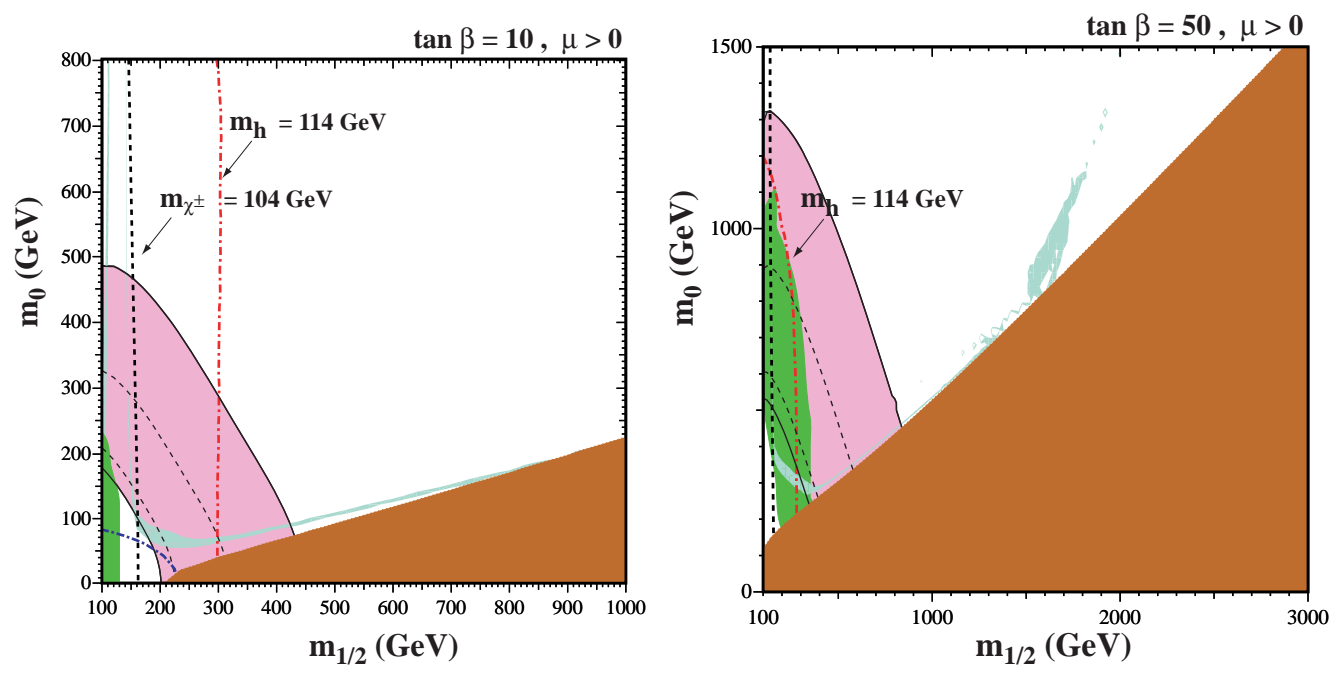

Figure 2: The $\left(m_{1 / 2}, m_{0}\right)$ planes for (a) $\tan \beta=10$ and $\mu>0$, assuming $A_{0}=0, m_{t}=175 \mathrm{GeV}$ and $m_{b}\left(m_{b}\right)_{S M}^{\overline{M S}}=4.25 \mathrm{GeV}$. The near-vertical (red) dot-dashed lines are the contours $m_{h}=$ $114 \mathrm{GeV}$, and the near-vertical (black) dashed line is the contour $m_{\chi^{ \pm}}=104 \mathrm{GeV}$. Also shown by the dot-dashed curve in the lower left is the corner excluded by the LEP bound of $m_{\tilde{e}}>99$ $\mathrm{GeV}$. The medium (dark green) shaded region is excluded by $b \rightarrow s \gamma$, and the light (turquoise) shaded area is the cosmologically preferred region. In the dark (brick red) shaded region, the LSP is the charged $\tilde{\tau}_{1}$. The region allowed by the E821 measurement of $a_{\mu}$ at the $2-\sigma$ level, is shaded (pink) and bounded by solid black lines, with dashed lines indicating the $1-\sigma$ ranges. In (b), $\tan \beta=50$.

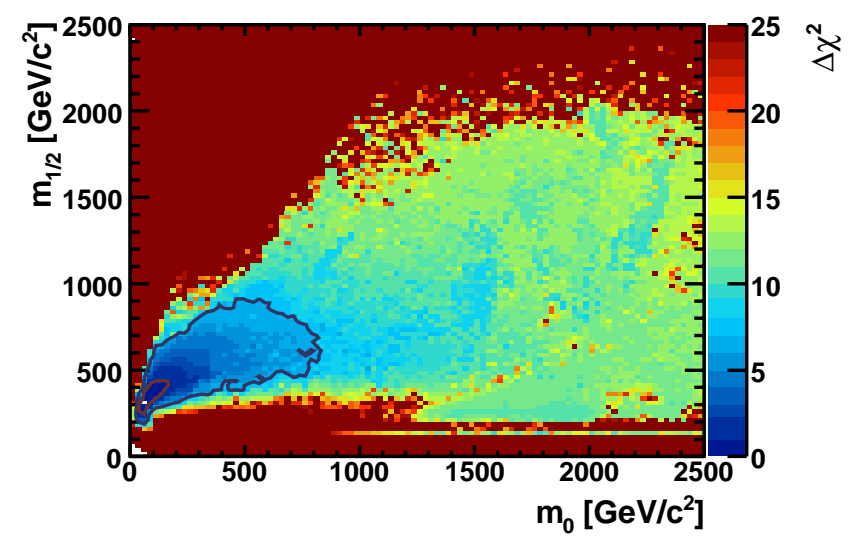

Figure 3: The $\Delta \chi^{2}$ functions in the $\left(m_{0}, m_{1 / 2}\right)$ planes for the CMSSM. We see that the coannihilation region at low $m_{0}$ and $m_{1 / 2}$ is favored. 

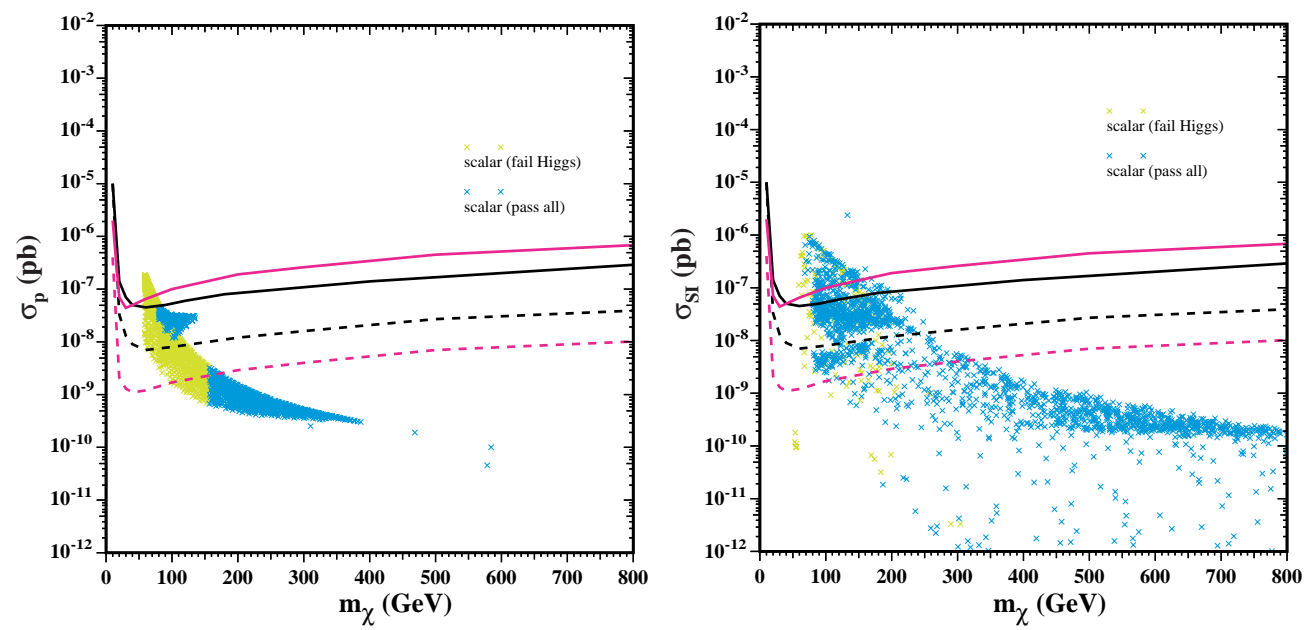

Figure 4: The neutralino-nucleon cross sections as functions of neutralino mass for the CMSSM with $\tan \beta=10$. Also shown are upper limits on the cross section from CDMS II [40] (solid black line) and XENON10 [41] (solid pink line), as well as the expected sensitivities for XENON100 [49] (dashed pink line) and SuperCDMS at the Soudan Mine [50] (dashed black line). Panel (b) shows the neutralino-nucleon cross sections as functions of neutralino mass for the CMSSM, with $5 \leq \tan \beta \leq 55,0 \leq m_{1 / 2} \leq 2000 \mathrm{GeV}, 100 \mathrm{GeV} \leq m_{0} \leq 2000 \mathrm{GeV}$, and $-3 m_{1 / 2} \leq A_{0} \leq 3 m_{1 / 2}$ We consider $\mu<0$ only for $\tan \beta<30$. Taken from [48].
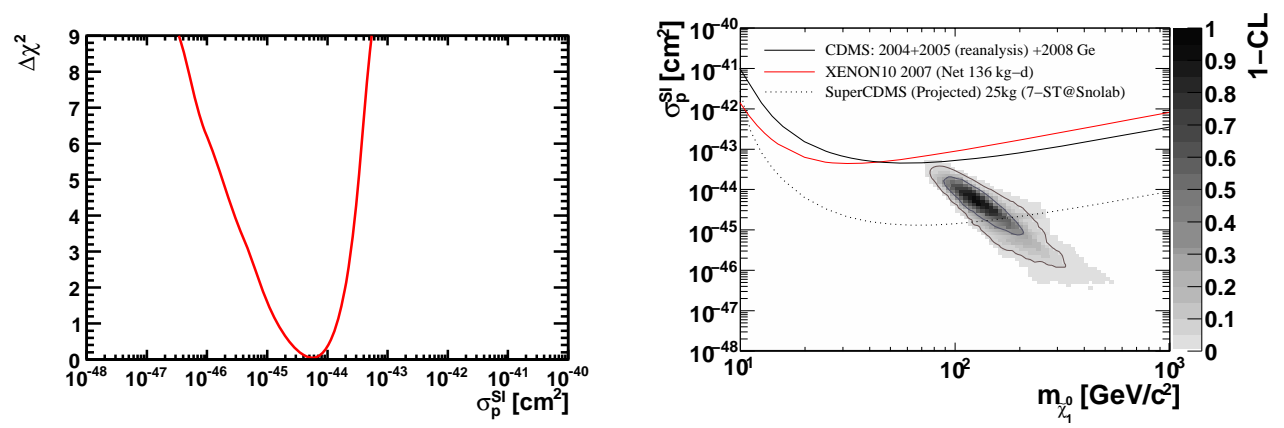

Figure 5: The likelihood functions for the spin-independent $\tilde{\chi}_{1}^{0}$-proton scattering cross section $\sigma_{p}^{\mathrm{SI}}\left(\right.$ in $\mathrm{cm}^{2}$ ) in the CMSSM (left panel). The correlation between the spin-independent DM scattering cross section $\sigma_{p}^{\mathrm{SI}}$ and $m_{\tilde{\chi}_{1}^{0}}$ in the CMSSM (right panel). 


\section{Discussion}

Cheng-Ju Lin (LBNL Berkely): What are the current constraints from the experimental bound on $B_{s} \rightarrow \mu^{+} \mu^{-}$on the CMSSM model?

Answer: The $B_{s} \rightarrow \mu^{+} \mu^{-}$constraint is essentially non-existent for low values of $\tan \beta$. They are somewhat more important for large tan beta. The current constraint on the branching ratio is $4.7 \times 10^{-8}$ At high $\tan \beta$, there are departures from the standard model value of $B_{s} \rightarrow \mu^{+} \mu^{-}\left(=3.4 \times 10^{-9}\right)$. The current constraint would exclude the lower left portion of the plane at $\tan \beta=50$ with $m_{0}<350 \mathrm{GeV}$ and $m_{1 / 2}<400 \mathrm{GeV}$. This region is also excluded by $b \rightarrow s \gamma$ as well as the LEP bound on the Higgs mass. It will become an intersting question as the experimental bound approaches the standard model value.

Guy Wormser (LAL Orsay): Could you comment on the recent press article mentioning the possibility of no dark energy at all?

Answer: I don't have too much to say on this. It would be attractive to have an alternative to dark energy. I don't know that it can be achieved consistently within the context of the concordance model known as $\Lambda \mathrm{CDM}$ which accounts for the growth of structure in the Universe and all of the other associated observations. It would be nice to get out of it, but I think currently we are stuck with the notion of dark energy or a cosmological constant.

Guy Wormser (LAL Orsay): A new BABAR result presented at this conference indicates a better agreement in the muon $g-2$ between theory and experiment. This would reduce room for new physics. How would it affect your constraints?

Answer: The new result has been examined and the combined result using $e^{+} e^{-}$and $\tau$ data has been derived in Davier et al. (arXiv:0908.4300 [hep-ph]) yielding yielding a deviation (in units of $10^{-10}$ ) of $24.6 \pm 0.8$ or a deviation of $3.1 \sigma$. This represents only a small change from the results I have presented.

The g-2 result drives one to the conclusion that sparticle masses are small (and hence favorable for the LHC), but this is not the only observable driving in that direction. The $\mathrm{W}$-mass also points to relatively low sparticle masses as well. If the g-2 result is weakened, the theory is certainly less constrained.

Vali Huseynov (Nakhchivan State University): You have reported about the dark matter candidates (neutralinos, axions, sneutrinos). Which of them is the best candidate for the cold dark matter? And, which one of them is the best candidate for the hot dark matter?

Answer: All of the candidates that I spoke about are cold dark matter candidates. The neutralino, snueutrino, and gravitino are all heavy particles which would be nonrelativistic at the time of structure formation. The axion though it is light is also a cold dark matter candidate. A standard candidate for hot dark matter is the light neutrino. But this is excluded as dark matter as the neutrino mass is constrained. In addition, hot dark matter produces too much structure on large scales and is no longer 
considered viable. 